\title{
VASCULAR AND INTERVENTIONAL RADIOLOGY
}

\section{Percutaneous placement of a biliary plastic stent}

\author{
Shyamkumar N Keshava, Suraj Mammen \\ Department of Radiodiagnosis, Christian Medical College, Vellore, Tamil Nadu, India
}

Correspondence: Dr. Shyamkumar N Keshava, Department of Radiodiagnosis, Christian Medical College, Vellore, Tamil Nadu, India. E-mail: aparna_shyam@yahoo.com

\begin{abstract}
Endoscopic biliary plastic stent placement is a well-established technique for unresectable and recurrent biliary obstructive disease and provides significant improvement in the quality of life. We describe a new technique of percutaneous placement of biliary plastic stent that has not been previously mentioned in the literature.
\end{abstract}

Key words: Biliary; drainage; plastic stent

\section{Introduction}

Endoscopic placement of plastic biliary stents for biliary obstruction is a well-established technique. ${ }^{[1,2]}$ Although percutaneous placement of stents at other sites is well known, ${ }^{[3]}$ as is the use of percutaneous placement of metallic biliary stents, ${ }^{[4]}$ we were unable to find a reference for percutaneous placement of plastic biliary stents in the literature.

\section{Case Report}

A 49-year-old man presented with jaundice of 3 months' duration and no clinical evidence of cholangitis. His bilirubin was $16 \mathrm{mg} / \mathrm{dl}$. MRI of the abdomen showed moderate intrahepatic and extrahepatic biliary dilatation; the common bile duct (CBD) was dilated up to the distal portion, with no obvious lesion. On endoscopy, a proliferative growth of the periampullary area was seen and a biopsy was taken from this. The histology was reported as moderately differentiated adenocarcinoma. A biliary drainage procedure was performed as described below. Ten

\begin{tabular}{|l|l|}
\hline \multicolumn{2}{|c|}{ Access this article online } \\
\hline Quick Response Code: & \\
\hline & Website: \\
\hline & www.ijri.org \\
\cline { 2 - 3 } & DOI: \\
\hline
\end{tabular}

days later, his bilirubin was $4 \mathrm{mg} / \mathrm{dl}$ and surgical treatment of the periampullary carcinoma is awaited.

\section{Technique}

Because the patient was sick, a two-stage procedure was performed. The procedure was performed in a digital subtraction angiography (DSA) suite (Siemens Multistar ${ }^{\circledR}$ T.O.P) under local anesthesia, with all aseptic precautions and antibiotic cover. USG guidance was used for the initial access with a 21G needle. A small amount of contrast medium was injected [Figure 1]. The tract was dilated and an $8 \mathrm{~F}$ external-internal biliary drainage was placed and connected to an external drainage bag.

Three days later, an internalization procedure was performed under local anesthesia. The site of obstruction was visualized after injecting contrast through the tube and a long 0.035 " guidewire was passed into the duodenum via the biliary drainage tube. After removal of the drainage tube a $7 \mathrm{~F} 35-\mathrm{cm}$ sheath [Figure 2] was inserted over the wire and advanced until the distal end projected into the duodenum across the stricture. A 7F $10-\mathrm{cm}$ plastic stent [Figure 3] Cotton-Leung ${ }^{\circledR}$ (Amsterdam) Sof-Flex ${ }^{\circledast}$ biliary stent (Cook Medical, USA) with a flap mechanism was passed over the wire into the sheath. The tapered distal few centimeters of the sheath dilator were cut transversely using a pair of scissors and the dilator was then used to advance the stent through the sheath till it was positioned at its tip [Figure 4]. Once the stent was located correctly, the sheath was withdrawn over the dilator, leaving the stent positioned across the biliary obstruction [Figure 5]. A 


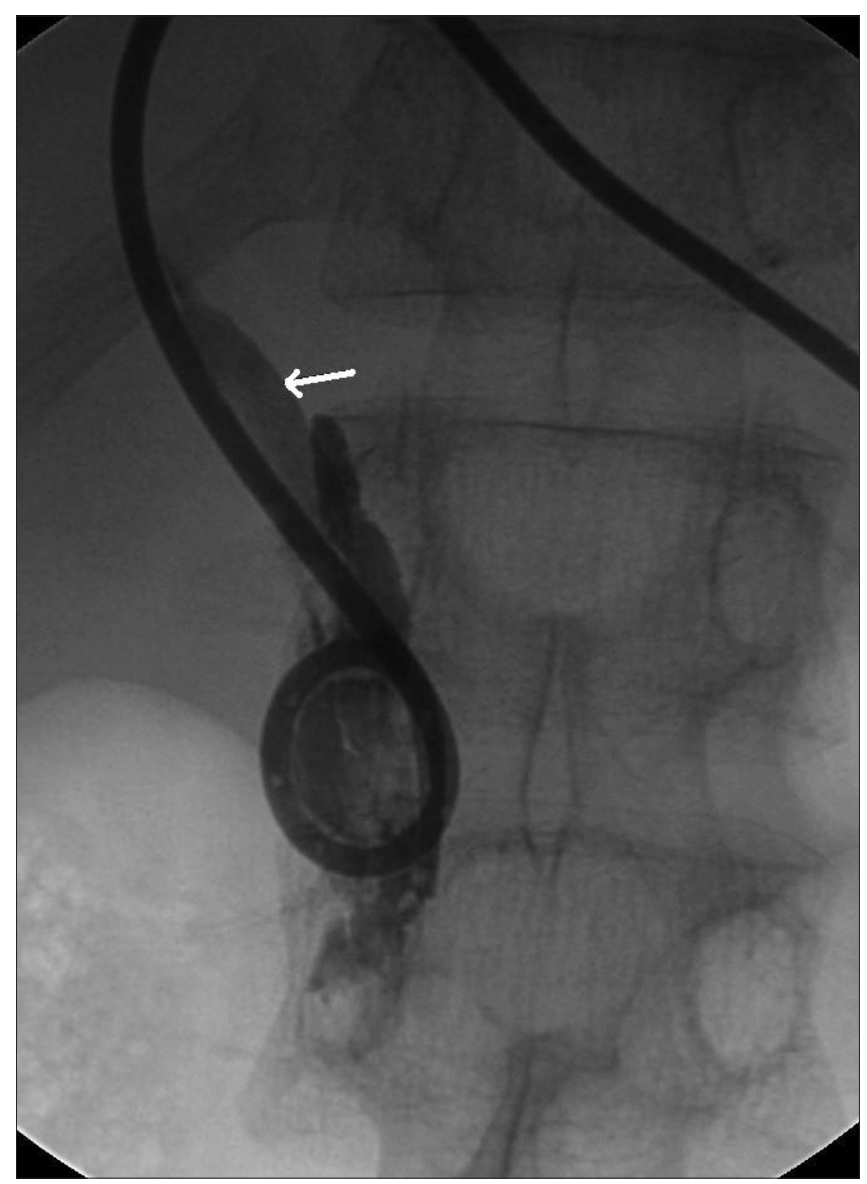

Figure 1: Frontal fluoroscopy spot radiograph shows opacification of the biliary system (arrow) after injection of contrast medium through the external biliary drainage tube

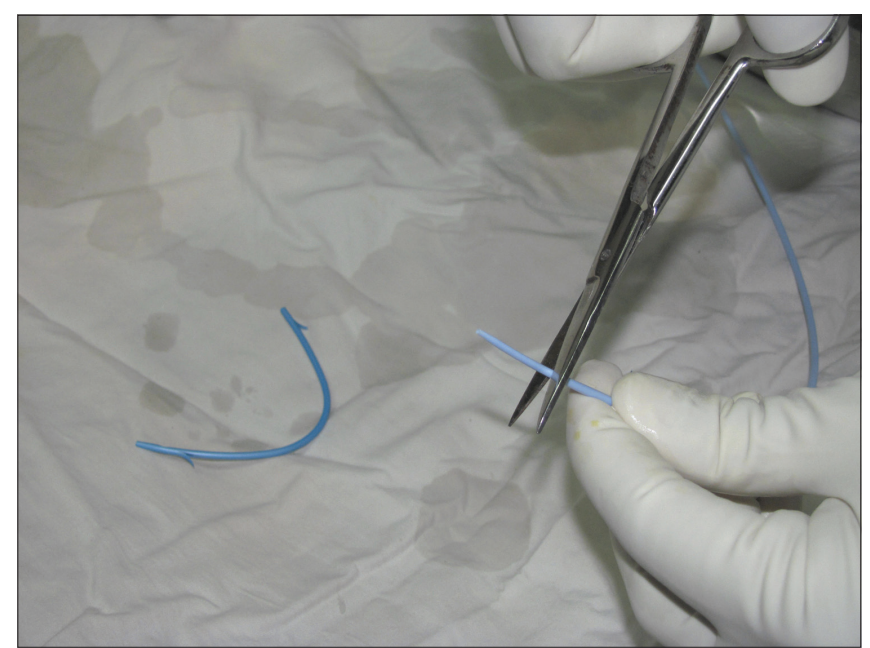

Figure 3: Cotton-Leung ${ }^{\circledR}$ (Amsterdam) Sof-Flex ${ }^{\circledR}$ biliary stent. The tapered terminal few centimeters of the sheath dilator was transversely cut using a pair of scissors

temporary external drainage tube (8F pigtail) was placed to prevent any clots from blocking the plastic stent, and this was removed after 2 days. The procedure was successful, with no complications.

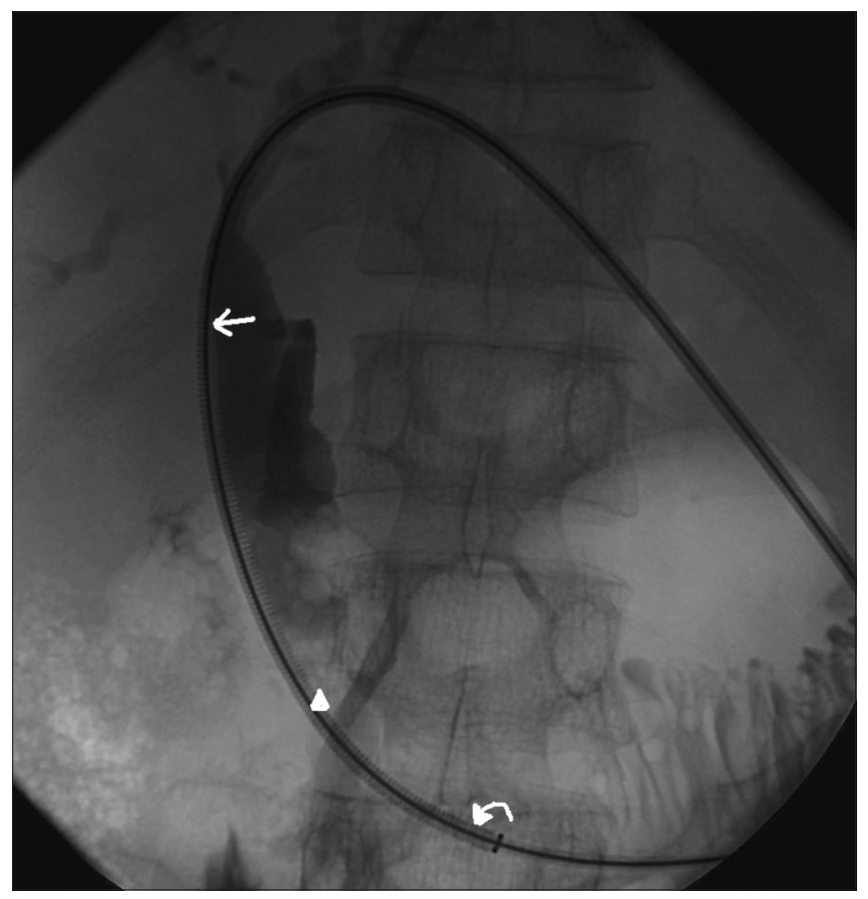

Figure 2: Frontal fluoroscopy spot radiograph shows a 7F 35-cm sheath (arrow) placed over the wire (arrowhead), with its distal end (curved arrow) protruding into the duodenum across the stricture

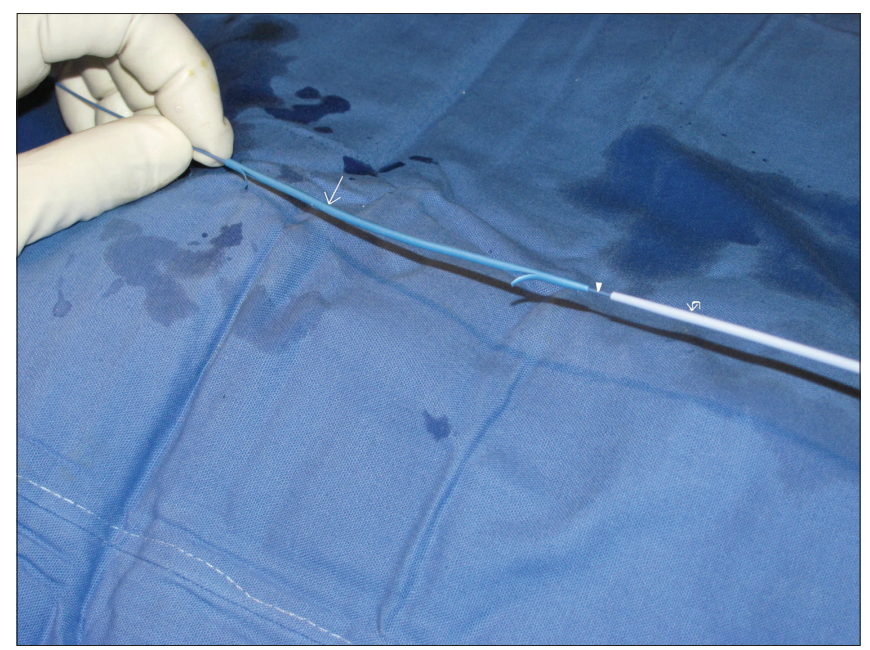

Figure 4: The plastic stent (arrow) is seen over the wire (arrowhead); it is followed by the dilator (curved arrow) as a pusher device

\section{Discussion}

We describe a simple technique for placing a biliary plastic stent percutaneously. The procedure can be performed in patients who require a plastic biliary stent for drainage but are not suitable for the endoscopic approach or when endoscopy is unsuccessful. Patients with ascites will require complete drainage of ascitic fluid prior to the procedure. The common designs to secure biliary stents in situ include flaps, pigtail, or mushroom. ${ }^{[5,6]}$ 


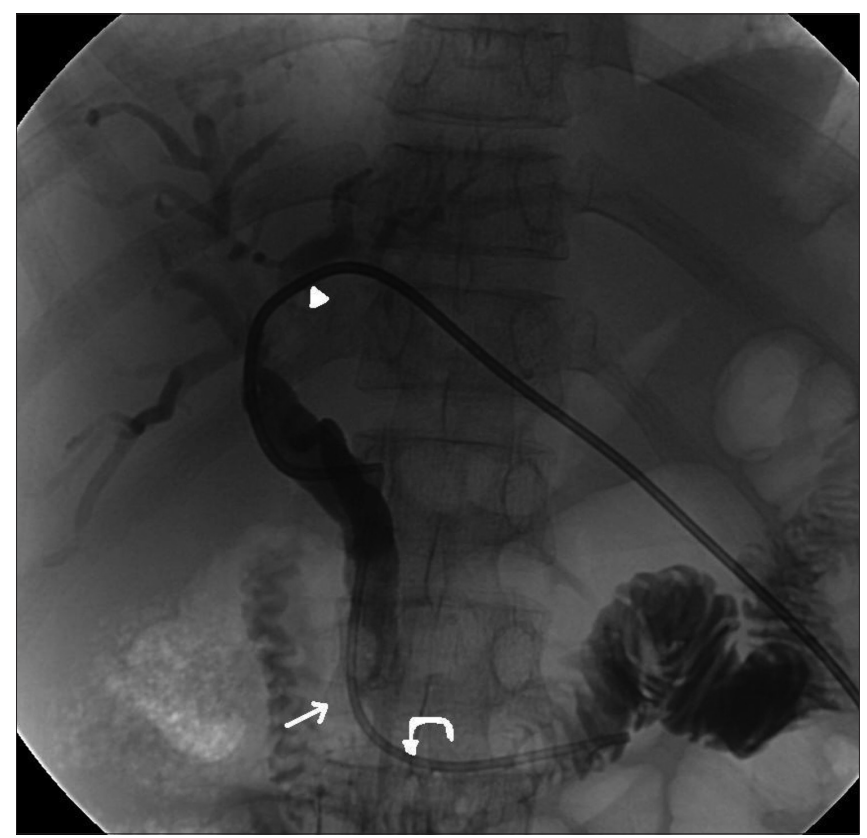

Figure 5: Frontal fluoroscopy spot radiograph shows the plastic stent (curved arrow) positioned across the stricture (arrow). A temporary $8 \mathrm{~F}$ external biliary drain (arrowhead) is also seen

The advantage of percutaneous placement of plastic stents when compared to endoscopic placement is that the patient can be kept in the supine position. We recommend that the sheath size be just large enough to take the stent so as to minimize liver surface trauma at the entry point. The tapered distal end of the dilator sheath should be removed to avoid its 'telescoping' into the stent. We have performed a similar procedure without any complications in five patients (three with malignant and two with benign pathology). All five had biliary obstruction due to strictures in the terminal CBD. Although we did not encounter any complications, potential complications include those related to percutaneous biliary drainage and misplacement of the stent. Compared to metal stents, the chances of blockage and migration of plastic stents are higher, and therefore plastic stents are perhaps best deployed in the short term, prior to definitive surgery; they may not be suitable for palliative therapy. An advantage with plastic stents is that they are very cheap compared to metal stents.

\section{Conclusions}

Though biliary plastic stents are usually placed through the endoscopic route, it is also possible to place them percutaneously. The procedure is safe and effective.

\section{Acknowledgements:}

Dr Tony Lamont

\section{References}

1. Van Boeckel PG, Vleggaar FP, Siersema PD. Plastic or metal stents for benign extra hepatic biliary strictures: A systematic review. BMC Gastroenterol 2009;9:96.

2. Yoon WJ, Ryu JK, Yang KY, Paik WH, Lee JK, Woo SM, et al. A comparison of metal and plastic stents for the relief of jaundice in unresectable malignant biliary obstruction in Korea: An emphasis on cost-effectiveness in a country with a low ERCP cost. Gastointest Endosc 2009;70:284-9.

3. Mitty HA, Train JS, Dan SJ. Placement of ureteral stents by antegrade and retrograde techniques. Radiol Clin North Am 1986;24:587-600.

4. Rossi P, Salvatori FM, Bezzi M, Maccioni F, Porcaro ML, Ricci P. Percutaneous management of benign biliary strictures with balloon dilation and self-expanding metallic stents. Cardiovasc Intervent Radiol 1990;13:231-9.

5. Englanda RE, Martina DF, Morris J, Sheridan MB, Frost R, Freemane A, et al. A prospective randomised multicentre trial comparing $10 \mathrm{Fr}$ Teflon Tannenbaum stents with $10 \mathrm{Fr}$ polyethylene Cotton-Leung stents in patients with malignant common duct strictures. Gut 2000;46:395-400.

6. Murai R, Hashiguchi F, Kusuyama A, Yoshimi M, Watanabe K, Okui $\mathrm{S}$, et al. Percutaneous stenting for malignant biliary stenosis. Surg Endosc 1991;5:140-2.

Cite this article as: Keshava SN, Mammen S. Percutaneous placement of a biliary plastic stent. Indian J Radiol Imaging 2011;21:231-3.

Source of Support: Nil, Conflict of Interest: None declared.

Staying in touch with the journal

1) Table of Contents (TOC) email alert

Receive an email alert containing the TOC when a new complete issue of the journal is made available online. To register for TOC alerts go to www.ijri.org/signup.asp.

\section{2) RSS feeds}

Really Simple Syndication (RSS) helps you to get alerts on new publication right on your desktop without going to the journal's website. You need a software (e.g. RSSReader, Feed Demon, FeedReader, My Yahoo!, NewsGator and NewzCrawler) to get advantage of this tool. RSS feeds can also be read through FireFox or Microsoft Outlook 2007. Once any of these small (and mostly free) software is installed, add www.ijri.org/rssfeed.asp as one of the feeds. 